Danilo Orlandini, Gualtiero de Bigontina Manuale di accreditamento delle strutture diabetologiche Terza edizione 
DANilo Orlandini • Gualtiero De Bigontina

\section{Manuale di accreditamento delle strutture diabetologiche}

Terza edizione

Springer 


\section{DANILO ORLANDINI}

Responsabile dello Staff Qualità Accreditamento

e dell'Unità Efficacia Clinica

dell'Azienda Unità Sanitaria Locale di Reggio Emilia

\section{GuALTiERo DE BigontinA}

Responsabile del Servizio di Diabetologia

dell'Ospedale di Pieve di Cadore

In copertina: La voix des airs. (C) René Magritte by Siae 2007

ISBN 978-88-470-0730-7

Springer-Verlag fa parte di Springer Science+Business Media

springer.com

(C) Associazione Medici Diabetologi (AMD) 2007

Quest'opera è protetta dalla legge sul diritto d'autore. Tutti i diritti, in particolare quelli relativi alla traduzione, alla ristampa, all'utilizzo di illustrazioni e tabelle, alla citazione orale, alla trasmissione radiofonica o televisiva, alla registrazione su microfilm o in database, $\mathrm{o}$ alla riproduzione in qualsiasi altra forma (stampata o elettronica) rimangono riservati anche nel caso di utilizzo parziale. La riproduzione di quest'opera, anche se parziale, è ammessa solo ed esclusivamente nei limiti stabiliti dalla legge sul diritto d'autore, ed è soggetta all'autorizzazione dell'editore. La violazione delle norme comporta le sanzioni previste dalla legge.

L'utilizzo in questa pubblicazione di denominazioni generiche, nomi commerciali, marchi registrati, ecc. anche se non specificatamente identificati, non implica che tali denominazioni o marchi non siano protetti dalle relative leggi e regolamenti.

Responsabilità legale per i prodotti: l'editore non può garantire l'esattezza delle indicazioni sui dosaggi e l'impiego dei prodotti menzionati nella presente opera. Il lettore dovrà di volta in volta verificarne l'esattezza consultando la bibliografia di pertinenza.

Copertina: Simona Colombo, Milano

Impaginazione: Graphostudio, Milano

Stampa: Arti Grafiche Nidasio, Assago

Stampato in Italia

Springer-Verlag Italia S.r.l., Via Decembrio 28, 20137 Milano 


\section{Presentazione}

Sono veramente lieto di presentare ai soci AMD (Associazione Medici Diabetologi) e alla comunità sanitaria italiana la nuova versione del Manuale di Accreditamento Professionale per le strutture di diabetologia: esso rappresenta una tappa importante e un'opportunità dell'evoluzione diabetologica.

Il manuale è il frutto del lavoro di un gruppo formato da Gualtiero de Bigontina e Danilo Orlandini. Il gruppo per la qualità in staff al Consiglio Direttivo Nazionale (CDN) si avvale attualmente dell'opera di Nino Cimino, Danila Fava, Illidio Meloncelli, e da una decina d'anni si occupa di miglioramento della Qualità dell'assistenza diabetologica.

A questo gruppo e a tutti coloro che si sono impegnati nella difficile strada della Qualità, i componenti di Agenzia Qualità che si sono via via avvicendati, gli operatori delle strutture di diabetologia che si sono accreditate in questi anni, i valutatori che hanno svolto le visite d'accreditamento, va il ringraziamento di AMD e mio personale.

Il progetto, nato con la presidenza di Claudio Noacco (1994), è stato una felice intuizione, il frutto di una lungimiranza che, superando molte difficoltà, ha continuato a diffondere tra i soci il concetto della Qualità e del Miglioramento. Dai concetti di accountability, di misurabilità e di miglioramento si sono generati progetti ugualmente importanti: l'Accreditamento Professionale tra pari, il Disease Management, gli Indicatori AMD, il File Dati AMD, gli Annali AMD.

Possiamo dire che la scommessa è stata vincente: il fatto di aver intuito l'importanza dei concetti della Qualità, di averli contestualizzati nel nostro mondo professionale, di averli fatti evolvere in funzione del contesto normativo e dell'utilità quotidiana, pone AMD e i suoi associati in una posizione privilegiata nel proprio contesto professionale e di fronte alle istituzioni.

Da parte mia e del CDN l'impegno a sostenere questa iniziativa e a continuare a farla crescere in modo che continui ad essere uno stimolo e un aiuto all'evoluzione della specie diabetologica. 
Un ringraziamento particolare alla Eli-Lilly Italia che in tutti questi anni, in partnership con AMD, ha sostenuto il progetto "Accreditamento Professionale in diabetologia".

Dott. Umberto Valentini

Presidente Nazionale Associazione Medici Diabetologi

Direttore UOC di Diabetologia per il Trattamento e l'Educazione Terapeutica Spedali Riuniti di Brescia 


\section{Indice}

Introduzione

\section{PARTE PRIMA}

\section{Linee guida per la revisione tra pari professionale} nelle strutture diabetologiche

1. Politica _ 15

2. Pianificazione 19

3. Comunicazione $\ldots$

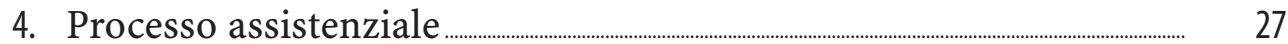

5. Educazione 31

6. Evidenze scientifiche, profili diagnostico-terapeutico-assistenziali,

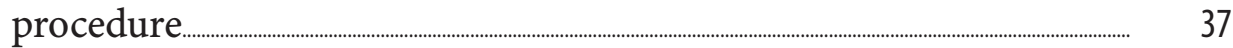

7. Sistema informativo .... _ _ 43

8. Verifica dei risultati e miglioramento _..._. 47

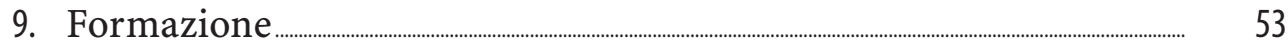

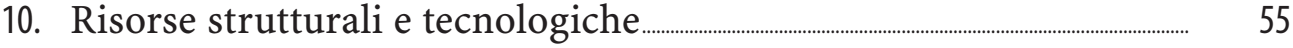

\section{PARTE SECONDA}

\section{Principi e criteri}

1. Principi fondamentali per la qualità professionale

2. Criteri 


\section{PARTE TERZA}

Lista di verifica per l'autovalutazione e la revisione tra pari professionale delle strutture diabetologiche

1. Principi fondamentali per la qualità professionale ....﹎.. 97

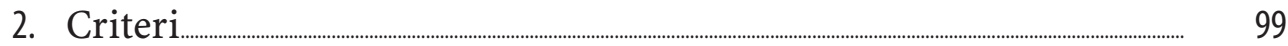

\section{PARTE QUARTA}

\section{Glossario e appendici}

1. Glossario del Manuale di Accreditamento dell'Associazione Medici Diabetologi.

2. Appendici 